EPJ Web of Conferences 38, 17007 (2012)

DOI: $10.1051 /$ epjconf/20123817007

(C) Owned by the authors, published by EDP Sciences, 2012

\title{
Problems of experimental and theoretical studies of the Gamow-Teller giant resonance in ${ }^{118} \mathrm{Sb}$
}

\author{
S. Yu. Igashov ${ }^{1, a}$, V.A. Rodin ${ }^{2}$ and M.H. Urin ${ }^{3}$ \\ ${ }^{1}$ All-Russia Research Institute of Automatics, Moscow, Russia \\ ${ }^{2}$ Institut für Theoretische Physik, Universität Tübingen, Tübingen, Germany \\ ${ }^{3}$ National Research Nuclear University "MEPhI", Moscow, Russia
}

\begin{abstract}
Properties of the Gamow-Teller giant resonance (GTGR) in ${ }^{118} \mathrm{Sb}$ are described within the semimicroscopic approach based on the extended version of the "charge-exchange" continuum-quasiparticlerandom-phase-approximation and phenomenological treatment of the spreading effect. The structure effect of the configuration splitting of this GTGR is confirmed. With taking this effect into account the experimental data on excitation of the GTGR in direct and resonance reactions are analyzed.
\end{abstract}

a e-mail: igashov@theor.mephi.ru

\section{Introduction}

Systematical experimental studies of the Gamow-Teller giant resonance in antimony isotopes by means of the direct charge-exchange ${ }^{112-124} \mathrm{Sn}\left({ }^{3} \mathrm{He}, t\right)$-reactions $[1,2]$ have been initially motivated by the theoretical prediction of the configuration splitting of the GTGR in antimony isotopes near $A=118$. The splitting was found within a simplified version of the "charge-exchange" continuumquasiparticle-RPA (pn-cQRPA) for some open-neutronshell nuclei [3]. This effect is due to filling the singleparticle neutron level, having the highest angular momentum for a given shell $\left(1 \mathrm{~h}_{11 / 2}\right.$ for tin isotopes). In experiments (see Refs. [1, 2]) the GTGR has been found as a bump, having no gross structure and the anomalously large total width (about $6 \mathrm{MeV}$ for isotopes near $\mathrm{A}=$ 118 ). Being studied in the same reaction, the GTGR in ${ }^{208} \mathrm{Bi}$ has the larger excitation energy and lesser total width $(3.7 \mathrm{MeV})$ [4]. The alternative method to observe the GTGR has been used in [5]. The method consisted in studying the excitation function of the resonance in

${ }^{117} \mathrm{Sn}\left(\mathrm{p}, \mathrm{n}_{\text {tot }}\right)$-reaction. Two rather narrow resonances (with the total width about $1 \mathrm{MeV}$ ) in this excitation function have been assigned to the GTGRs excited in the compound-nucleus ${ }^{118} \mathrm{Sb}$ and built on the ground and two-quasiparticle $\left(d_{3 / 2}, s_{1 / 2}^{-1}\right)$ states of the parent nucleus ${ }^{118} \mathrm{Sn}$. The partial (elastic) proton-escape width has been also deduced for both GTGRs [5]. These experiments are planned to be continued [6]. Recently, the extended version of the pn-cQRPA was developed and applied to study the Gamow-Teller $2 v \beta \beta$-decay amplitudes for open-shell nuclei [7]. Using this method as a base, in the present work we: (i) revise the previous study of the configuration splitting of the GTGR; (ii) analyze rather contradictive experimental data about the GTGR(s) in ${ }^{118} \mathrm{Sb}$; (iii) formulate the open problems for further experimental and theoretical studies. The more detailed version of this work, where other antimony isotopes near $A=118$ are also considered, will be published soon [8]

\section{Description of the GTGR in ${ }^{118} \mathrm{Sb}$ within the pn-cQRPA}

Within the cQRPA the Landau damping and singleparticle continuum are taken into account, while the spreading effect is ignored. A phenomenological partially self-consistent mean field and realistic interactions in the particle-hole ( $p-h)$ and particle-particle ( $p-p)$ channels are used in [7], as the input quantities for calculations of various "charge-exchange" strength functions within the pn-cQRPA. Using the same input quantities, we take the model parameters, as described in [7]. In particular, we use the mean-field geometrical parameter $r_{0}=1.21 \mathrm{fm}$ [9]. Other parameters are taken from independent data, except for the strength $g^{\prime}$ of the spin-isospin part of the Landau-Migdal $\mathrm{p}$-h interaction. This parameter is adjusted to reproduce in calculations of the GT strength function $S_{G T}^{(-)}\left(E_{x}\right) \quad\left(E_{x}\right.$ is the excitation energy) the centroid of the energy $E_{x}^{\text {c.e. }}=10.6 \mathrm{MeV}$ deduced in [2] 
for the GTGR in ${ }^{118} \mathrm{Sb}$. The calculated GT strength function exhibits the splitting of the GTGR into two components, having the energy $E_{x,<}=9.29 \mathrm{MeV}$, $E_{x,>}=12.11 \mathrm{MeV}$ and the total ("natural" proton-escape) width $\Gamma_{<}^{\uparrow}=5 \mathrm{keV}, \quad \Gamma_{>}^{\uparrow}=81 \mathrm{keV}$, respectively. Both components in aggregate exhaust about $73 \%$ of the Ikeda sum rule, while their relative strength $B_{<}(G T) / B_{>}(G T)$ equals to 1.31 . In such a way we confirm the configuration splitting of the GTGR in ${ }^{118} \mathrm{Sb}$ predicted in [3] within the simplified version of the pn-cQRPA, in which the charge-exchange part of the $p$-p interaction was not taken into account. Using the obtained calculation results, one can understand some of experimental data obtained in [5]. The GTGR high-energy component $\left(\mathrm{GTGR}_{>}\right)$can be identified with the observed low-energy resonance $\mathrm{GTGR}_{1}$, having the energy $E_{x, r_{1}}=12.06 \mathrm{MeV}$.

The partial (elastic) proton-escape width $\Gamma_{p_{0}, r_{1}}^{\uparrow}=56 \mathrm{keV}$ of this resonance has the same order of magnitude, as the calculated total "natural" proton-escape width of GTGR $>$. Non-observation of the resonance, corresponding to the GTGR $_{<}$, can be explained by a small value of the partial (elastic) proton-escape width of this resonance, as it takes place in applying to the calculated total "natural" width.

\section{Phenomenological description of the spreading effect}

Such description within the pn-cQRPA-based method includes the statistical assumption: after energy averaging each two-quasiparticle-type excitation (with a given set of quantum numbers) "decays" into chaotic states independently of one another. We take the spreading effect approximately into account in terms of the energydependent spreading width $\Gamma^{\downarrow}\left(E_{x}\right)$ used for each resonance with the energy $E_{x}$ found within pn-cQRPA:

$$
\begin{aligned}
& \Gamma^{\downarrow}\left(E_{x}>\Delta\right)=\Gamma_{0} \frac{e_{x}^{2}}{1+e_{x}^{2}}, \quad e_{x}=\frac{E_{x}-\Delta}{B}, \\
& \Gamma^{\downarrow}\left(E_{x} \leq \Delta\right)=0 .
\end{aligned}
$$

The "gap" parameter $\Delta=3 \mathrm{MeV}$ and the "saturation" parameter $B=7 \mathrm{MeV}$ are taken from semi-microscopic description of various giant resonances in closed-shell nuclei $[10,11]$, while $\Gamma_{0}$ is an adjustable parameter. Applying Eq. (1) to the GTGR in ${ }^{208} \mathrm{Bi}$ studied in [4], one can get the parameter $\Gamma_{0}=5 \mathrm{MeV}$. To continue further identification of the GTGR $>$ with the GTGR G $_{1}$ observed in [5], we take the parameter $\Gamma_{0}=1.6 \mathrm{MeV}$ in evaluation of the energy-averaged GT strength function (figure 1). As a result, we get two-bump GTGR with the GTGR $>$, having the observed total width $\Gamma_{>}=1 \mathrm{MeV}$ [5]. This is in contrast with observation of [2]. To get the GTGR without gross structure, we choose the parameter $\Gamma_{0}=8$ $\mathrm{MeV}$. In such a case, the calculated energy-averaged GT strength function (figure 2) exhibits the one-bump GTGR, having the observed in [2] total width $\Gamma \approx 6$
$\mathrm{MeV}$. This GTGR is a superimposition of the GTGR $<$ and GTGR > also shown in figure 2 .

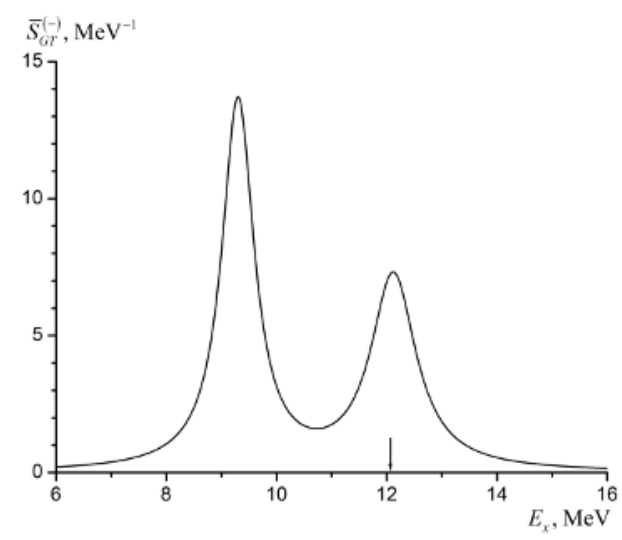

Fig. 1. The energy-averaged strength function calculated for the GTGR built on the ground state of ${ }^{118} \mathrm{Sn}$ with taking the adjustable parameter $\Gamma_{0}=1.6 \mathrm{MeV}$ in Eq. (1). The arrow indicates the energy of the $\mathrm{GTGR}_{1}$ found in [5].

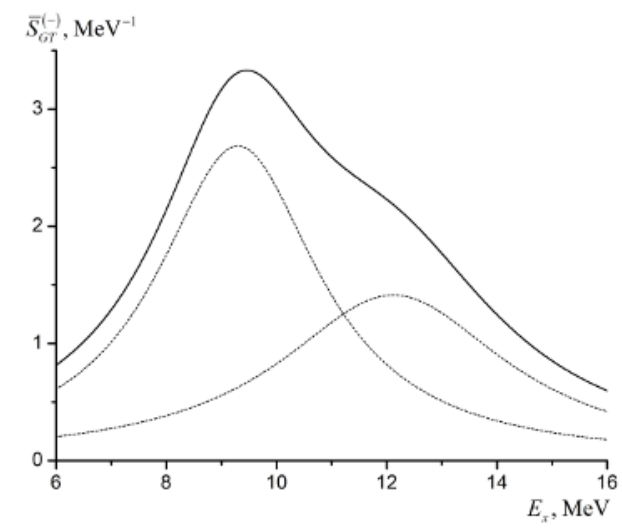

Fig. 2. The same as in figure 1 , but with $\Gamma_{0}=8 \mathrm{MeV}$. The strength functions related to the $\mathrm{GTGR}_{<}$and $\mathrm{GTGR}_{>}$are also shown.

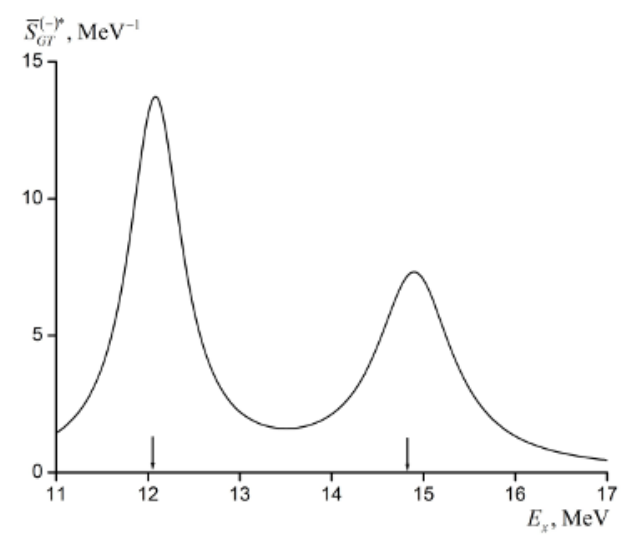

Fig. 3. The same as in figure 1, but for the GTGR built on the $\left(d_{3 / 2}, s_{1 / 2}^{-1}\right)$ excited state of ${ }^{118} \mathrm{Sn}$. The arrows indicate the energy of the GTGRs found in [5]. 
The choice of the simple low-energy excited state $\left(d_{3 / 2}\right.$, $s_{1 / 2}^{-1}$ ) of the parent nucleus ${ }^{118} \mathrm{Sn}$ among other low-energy states is explained by expectation of a large elastic proton-escape width of the GTGR ${ }^{*}$ built on the mentioned state. Within the used calculation scheme the energy of this state is found to be $E_{x}^{*}=2.78 \mathrm{MeV}$. In accordance with the known Brink hypothesis, for the corresponding GT strength function we have

$\bar{S}_{G T}^{(-)^{*}}\left(E_{x}\right)=\bar{S}_{G T}^{(-)}\left(E_{x}+E_{x}^{*}\right)$.

Taking $\bar{S}_{G T}^{(-)}\left(E_{x}\right)$ in this equation from figure 1, we get the two-bump GTGR * shown figure 3. The calculated energy of the GTGR ${ }_{>}^{*}, E_{x,>}^{*}=14.89 \mathrm{MeV}$, is close to the observed in [4] energy of $\mathrm{GTGR}_{2}, E_{x, r_{2}}=14.82 \mathrm{MeV}$. It is interesting to note, that the calculated energy of the $\mathrm{GTGR}_{<}^{*}, E_{x,<}^{*}=12.08 \mathrm{MeV}$, is close to the experimental energy of the GTGR ${ }_{1}$. The reason for this exotic situation consists in proximity of the splitting energy and energy of the above-mentioned two-quasiparticle state within the used calculation scheme. The possibility to observe the GTGR * depends on the value of the corresponding elastic proton-escape width, which is expected to be small, as compared with that for the GTGR ${ }^{*}$ or $\left(\mathrm{GTGR}_{2}\right)$.

\section{Conclusions}

Being based on the extended version of the pn-cQRPA, the performed analysis of rather contradictive experimental data on excitation of the GTGR(s) in ${ }^{118} \mathrm{Sb}$ allows us to make the following conclusions. (i) The configuration splitting effect is responsible (at least partially) for the anomalously large total width of the one-bump GTGR observed in the direct ${ }^{118} \mathrm{Sn}\left({ }^{3} \mathrm{He}, \mathrm{t}\right)$ reaction. (ii) This effect allows one to understand nature and to describe the energy of the two GTGRs observed in the resonance ${ }^{117} \mathrm{Sn}\left(\mathrm{p}, \mathrm{n}_{\mathrm{tot}}\right)$-reaction (one can also estimate qualitatively the elastic proton-escape of the low-energy GTGR). The main challenge in this experimental study is observation of the anomalously small total width of the above-mentioned GTGRs. Confirmation of this observation would be evidence of partial conservation of the spin-isospin $\mathrm{SU}(4)$-symmetry, which leads to suppression of the spreading effect. For the abovediscussed reasons continuation of experimental studies of the GTGR by means of the resonance ( $\left.\mathrm{p}, \mathrm{n}_{\mathrm{tot}}\right)$-reaction seems to be necessary. Further theoretical studies consist in extension of the pn-cQRPA-based method to describe: (i) direct proton decay of the GTGR built on the ground state of the parent nucleus (for closed-shell parent nuclei the corresponding method is formulated recently [12]); (ii) the spreading effect in terms of an effective singleparticle optical-model potential (in applying to closedshell nuclei it is done within the semi-microscopic approach of Refs. [10, 11]; (iii) in a microscopic way the GTGRs built on two-quasiparticle states of a parent nucleus (instead of using the Brink hypothesis).
This work is partially supported by the Russian Fund for Basic Research under grant no. 12-02-01303-a.

\section{References}

1. J. Janecke, et al., Phys. Rev. C 48, 2828 (1993)

2. K. Pham, et al., Phys. Rev. C 51, 526 (1995)

3. V.G. Guba, M.A. Nikolaev, M.G. Urin, Phys. Lett. B 218, 283 (1989)

4. H. Akimune et al., Phys. Rev. C 52, 604 (1995)

5. B. Ya. Guzhovskii, B. M. Dzyuba, and V. N. Protopopov, JETP Lett. 40, 1322 (1984)

6. S. N. Abramovich, private communication.

7. S. Yu. Igashov, Vadim Rodin, Amand Faessler, and M. H. Urin, Phys. Rev. C 83, 044301 (2011)

8. S.Yu. Igashov, V.A. Rodin, and M.H. Urin, Phys. At. Nucl. (in press)

9. M. L. Gorelik and M.G. Urin, Bull. RAS, Physics, 76, 863 (2012)

10. M.H. Urin, Nucl. Phys. A 811, 107 (2008)

11. M.H. Urin, Phys. At. Nucl. 74, 1189 (2011)

12. I.V. Safonov and M.H. Urin, Phys. At. Nucl. 75 (2012) (in press) 